\title{
14-3-3 $\gamma$-isoform detection distinguishes sporadic Creutzfeldt-Jakob disease from other dementias
}

\author{
B R J Van Everbroeck, J Boons, P Cras
}

J Neurol Neurosurg Psychiatry 2005;76:100-102. doi: 10.1136/jnnp.2003.032037

We developed a polyclonal antiserum directed to the $\gamma$ isoform of the human 14-3-3 protein and compared the immunoreactivity with a commercially available antibody (CG31). We analysed 14-3-3 in 253 cerebrospinal fluid samples blinded for the diagnosis by western blot and ELISA, with a commonly used polyclonal antiserum (Sc-731) and the $\gamma$ specific antibodies. Our patient population consisted of 52 patients with definite sporadic Creutzfeldt-Jakob disease (sCJD) and 201 patients with a different final diagnosis. We obtained similar sensitivity, ranging from $96 \%$ to $98 \%$ with all antibodies. Of all the samples that were false positive with Sc-731, 50\% were negative with both $\gamma$-isoform specific antibodies resulting in a significantly higher specificity $185 \% \mathrm{v}$ $93 \%$, respectively). If only sCJD and patients with dementia differing from SCJD were analysed we found that $64 \%$ of false positives were negative which also resulted in significantly increased specificity and positive predictive value.

The $\gamma$-isoform specific antibodies strongly improve the specificity of the immunoblot and might improve worldwide acceptance of the use of the 14-3-3 assay in the differential diagnosis of $\mathrm{SCJD}$.

$\mathrm{T}$ he commonest human prion disease is sporadic Creutzfeldt-Jakob disease (sCJD), with an approximate incidence of one patient per million inhabitants per year. Definite diagnosis of sCJD requires neuropathological study. ${ }^{1}$ For ante mortem diagnosis, a positive cerebrospinal fluid (CSF) 14-3-3 assay was added to the criteria for "probable" sCJD by the World Health Organization (WHO). ${ }^{2}$ Although numerous studies ${ }^{3-8}$ have reported the importance of the 143-3 assay in the diagnosis of sCJD, other studies claim it is not sufficiently specific in large populations to be used as a true diagnostic test. ${ }^{9}{ }^{10}$ False positive results were obtained in patients with acute or subacute neuronal destruction and in some patients with dementia. ${ }^{11}$ False negative results were described mainly in early stages or in SCJD patients with a long disease duration. The interpretation of the assay and the number of false positives continue to be a matter of discussion.

The 14-3-3 protein has a molecular weight of $30 \mathrm{kDa}$ and seven known isoforms $(\beta, \epsilon, \gamma, \eta, \tau$, and $\varphi){ }^{{ }^{12}}$ The isoforms $\beta$, $\epsilon, \gamma$, and $\eta$ have been identified in the CSF of patients with SCJD. ${ }^{513}$

The aim of this study was to investigate whether antibodies directed specifically against the $\gamma$-isoform could improve the differential diagnosis. We analysed the sensitivity and specificity by using the new antibodies in a population of 52 sCJD and 201 control patients in whom a definite diagnosis had previously been made. Next, we investigated whether a quantitative enzyme linked immunoassay (ELISA) would improve our results compared with the subjective western blot technique.

\section{MATERIALS AND METHODS}

Our laboratory performs the 14-3-3 analyses in CSF for the whole of Belgium. When a clinician suspects SCJD a CSF sample and all relevant clinical data are sent to us. We study the clinical data, carry out the 14-3-3 assay, and discuss the result with the neurologist. Afterwards the clinical status of the patient is followed up. After death or after 6-12 months the clinician reports the final diagnosis, and, if applicable, a neuropathological investigation is done.

Since 1998, we have analysed CSF samples of 393 patients diagnosed as having "possible" sCJD. ${ }^{11}$ For 261 (66\%) tested patients a final diagnosis could be made using clinical or neuropathological follow up data. We further excluded three patients with a hereditary prion disease and five patients with SCJD who remained "probable" due to lack of neuropathological confirmation. Thus our final study population consisted of 253 patients. In this series three distinct groups of patients could be identified: patients with SCJD $(\mathrm{n}=52)$, patients with dementia differing from sCJD (OD, $\mathrm{n}=140$ ), and patients with other neurological disorders (OND, $\mathrm{n}=61$ ) (table 1).

The standard 14-3-3 assay was performed as previously described..$^{414}$ Blots were incubated with Sc-731 (Santa Cruz Inc, Santa Cruz, CA), which is directed against the $\gamma$-isoform but recognises multiple isoforms. ${ }^{13}$ Each blot included a human brain homogenate ( $0.1 \mathrm{mg}$ of total protein) as positive control and CSF of a hydrocephalic patient as negative control. A sample was considered to be positive when the immunoblot showed a reactive band of electrophoretic mobility similar to the positive control. All CSF samples were tested twice and if contradictory, a third test was done for final evaluation.

We analysed the amino acid (AA) sequences of all 14-3-3 isoforms using the Swiss Prot data (http://us.expasy.org/ sprot/) and selected $2 \gamma$ specific epitopes: AA 2-12 and 235245 . Both peptides were injected into the same rabbit and a $\mathrm{pAb}$ mix generated. The obtained antisera were purified by affinity chromatography using the initial peptides. Analysis showed an optimal signal of the $\gamma$ 14-3-3 pAb mix at a 1:500 dilution. We further analysed a recently available commercial monoclonal antibody (mAb) and obtained optimal results with 1:750 dilution (CG31, Biocarta Inc, Hamburg, Germany). Next we examined the day to day variance on eight samples (four definite sCJD, two Alzheimer's disease $(A D)$, one dementia with Lewy bodies and one multiple sclerosis) on three consecutive days and one week later. The visual analysis by two independent observers

Abbreviations: $A A$, amino acid; $A U$, arbitrary unit; $C S F$, cerebrospinal fluid; ELISA, enzyme linked immunoassay; FP, false positives; OD, patients with dementia differing from SCJD; OND, other neurological disorders; sCJD, sporadic Creutzfeldt-Jakob disease; TP, true positives 


\begin{tabular}{|c|c|c|c|c|c|c|c|c|}
\hline \multirow[b]{2}{*}{ Diagnosis } & \multirow[b]{2}{*}{$\mathrm{N}$} & \multicolumn{2}{|c|}{ Sc-731 } & \multicolumn{2}{|c|}{ CG31 } & \multicolumn{2}{|c|}{$\gamma$ 14-3-3 } & \multirow[b]{2}{*}{$p$ value } \\
\hline & & Pos & Neg & Pos & Neg & Pos & $\mathrm{Neg}$ & \\
\hline $\mathrm{sCJD}$ & 52 & 51 & 1 & 50 & 2 & 50 & 2 & \\
\hline Sensitivity (\%) & & 98 & & 96 & & 96 & & $>0.1$ \\
\hline Controls & 201 & 30 & 171 & 16 & 185 & 15 & 186 & \\
\hline Specificity (\%) & & 85 & & 92 & & 93 & & 0.04 \\
\hline $\operatorname{PPV}(\%)$ & & 63 & & 76 & & 77 & & 0.09 \\
\hline$O D$ & 140 & 14 & 126 & 5 & 135 & 5 & 135 & \\
\hline Frontotemporal dementia & 9 & 0 & 9 & 0 & 9 & 0 & 9 & \\
\hline Alzheimer's disease & 75 & 5 & 69 & 1 & 34 & 1 & 34 & \\
\hline Vascular dementia & 29 & 4 & 25 & 2 & 16 & 2 & 16 & \\
\hline Lewy body dementia & 28 & 5 & 23 & 2 & 15 & 2 & 15 & \\
\hline Specificity (\%) & & 90 & & 96 & & 96 & & 0.03 \\
\hline PPV (\%) & & 78 & & 91 & & 91 & & 0.05 \\
\hline OND & 61 & 16 & 45 & 11 & 50 & 10 & 51 & \\
\hline Multiple system atrophy & 5 & 0 & 5 & 0 & 5 & 0 & 5 & \\
\hline Psychiatric disorders & 11 & 1 & 10 & 0 & 11 & 0 & 11 & \\
\hline Hashimoto's encephalitis & 5 & 1 & 4 & 0 & 5 & 0 & 5 & \\
\hline ALS & 2 & 1 & 1 & 0 & 2 & 0 & 2 & \\
\hline Paraneoplastic syndrome & 12 & 4 & 8 & 3 & 9 & 3 & 9 & \\
\hline Viral encephalopathy & 15 & 5 & 10 & 4 & 11 & 4 & 11 & \\
\hline Metabolic encephalopathy & 11 & 4 & 7 & 4 & 7 & 3 & 8 & \\
\hline Specificity (\%) & & 74 & & 82 & & 84 & & 0.19 \\
\hline PPV (\%) & & 76 & & 82 & & 82 & & $>0.1$ \\
\hline \multicolumn{9}{|c|}{$\begin{array}{l}\text { *We used three different antibodies to analyse 14-3-3: Sc-731 is the standard polyclonal antisera, } \gamma \text { 14-3-3 ( } \gamma \\
\text { directed polyclonal antisera) and the CG31 Biocarta monoclonal antibody. Calculation of the sensitivity, specificity, } \\
\text { and PPV of 14-3-3 detection with regard to sCJD diagnosis. } \\
\text { ALS, amyotrophic lateral sclerosis; Neg, negative; Pos, positive band; PPV, positive predictive value. }\end{array}$} \\
\hline
\end{tabular}

resulted in the same evaluation on all occasions. Combinations of these antibodies were studied in a sandwich ELISA. The intra- and interassay precision of the ELISA were always below 10\%. For both western blot and ELISA we used the same brain homogenate as positive standard.

\section{RESULTS}

We analysed 253 CSF samples for the presence of 14-3-3 using three different antibodies. Using Sc-731 pAb we identified 14-3-3 in 81 samples of which 51 were true positives (TP) and 30 false positives (FP), which meant the final diagnosis was other than sCJD. Applying CG31 we found $50 \mathrm{TP}$ and $16 \mathrm{FP}$ while the $\gamma$ 14-3-3 pAb resulted in 50 TP and 15 FP (table 1). We identified similar sensitivity but significantly increased specificity with both the CG31 mAb and the $\gamma$ 14-3-3 pAb when compared with the Sc-731 pAb ( $85 \% \vee 92 \%$, respectively table 1$)$.

In the OD population we found that nine out of 14 bands (64\%) identified with Sc-731 did not show any immunoreactivity with either $\gamma$ specific antibody resulting in an increased specificity and positive predictive value (table 1 ). In the OND group we found that five out of 16 bands (31\%) were negative with both $\gamma$ specific antibodies compared with Sc-731. This improvement did not result in a significantly increased specificity ( $74 \% \vee 84 \%$, respectively, table 1$)$.

In some immunoblots faint bands are visible but are scored as negative (fig l, lane 4), which indicates the difficulty of using a subjective immunoblot. We therefore developed a "quantitative" sandwich ELISA technique.

The optical density of the positive standard, a brain homogenate, on the ELISA was equalised to 1 arbitrary unit $(\mathrm{AU})$ in all tests. The best signal to noise ratio and standard curve were obtained when the Sc-731 pAb was used for capture and CG31 mAb for detection. We identified a median level of 1.00 AU 14-3-3 protein in SCJD patients using the ELISA (range 0.23-1.96 AU). In the patients with a different final diagnosis we found a median of 0.23 AU (range 0.07$1.90 \mathrm{AU})$. The results from both groups were significantly different with the Mann-Whitney U test $(p<0.0001)$. In the OD patients a median of $0.18 \mathrm{AU}$ (range $0.10-1.60 \mathrm{AU}$ ) was found and in the OND patients a median of $0.23 \mathrm{AU}$ (range 0.07-1.90 AU). The results of both these groups differed significantly from the sCJD group $(\mathrm{p}<0.0001)$, however, there was no significant difference between the two groups.

An optimal cut-off value for diagnostic use of $0.28 \mathrm{AU}$ was suggested by a receiver operating characteristics analysis. Using this cut-off we obtained $94 \%$ sensitivity. We found specificity of $85 \%$ in the OND group, $96 \%$ in the dementia group, and an overall average of $93 \%$. These results were not different from those obtained by the subjective $\gamma$-isoform specific western blot assay.

\section{DISCUSSION}

We found that $\gamma$-isoform directed antibodies significantly increase the specificity of the 14-3-3 assay without decreasing the sensitivity especially in patients with a different type of dementia. This was further supported by the finding that the differential diagnostic significance was unchanged by using a quantitative ELISA assay compared with a subjective immunoblot technique.

The results of this and our previous studies ${ }^{4}{ }^{15}$ are very similar to those of other European labs reporting a high sensitivity and specificity $(>85 \%) .{ }^{3-8}$ On the other hand, some Asian and American groups have reported low specificity with similar technology. ${ }^{10} 1617$ These differences are most likely due to the criteria used for sample selection. Our results clearly indicate that some neurological disorders which can temporarily mimic sCJD, such as vascular, infectious, and inflammatory encephalopathies may result in false positives and should be excluded before analysis (table 1).

Another pitfall in the 14-3-3 analysis is the existence of weak bands which are regarded as positive and faint bands which are regarded as negative if their signal was identical on all three examined blots. Previously, we showed that densitometric analysis, compared with visual analysis, of 14-3-3 leads to increased specificity and lower sensitivity. ${ }^{4}$ Therefore, this technique was not further explored. In this study we described an ELISA technique, which has similar specificity and could be a valuable alternative for the western blot. ${ }^{4}$ 


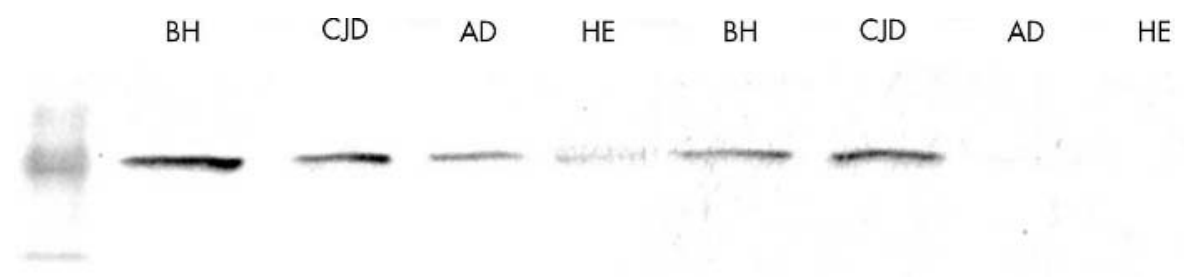

Figure 1 Western blot detection of 14-3-3 protein using Sc-731 pAb (lanes 1-4) and CC31 mAb (lanes 5-8). The molecular marker is depicted on the left side (end). The bands of the positive control (BH, brain homogenate) and the patient with CJD (CJD) are always scored as positive. With the Sc$731 \mathrm{pAb}$ the sample from a patient with Alzheimer's disease (AD) is positive and the sample from the patient with Hashimoto's encephalitis (HE), which resulted in only a very faint band, is scored as negative. With the CG31 mAb the AD and HE samples are negative.

Recent studies have also shown that the measurement of tau protein and $A \beta_{1-42}$ in CSF has gained acceptance in the differential diagnosis of sCJD. ${ }^{11}{ }^{15}$ Unlike $A \beta_{1-42}$, which is normal or decreased in SCJD, very high tau protein levels can point the diagnosis towards SCJD. ${ }^{14}$ 18

The combination of clinical data correlation and the availability of the novel $\gamma$-isoform specific antibodies might improve worldwide acceptance of the use of the 14-3-3 assay in the differential diagnosis of SCJD.

\section{ACKNOWLEDGEMENTS}

The authors thank all the physicians who sent CSF samples and clinical information of patients with suspected SCJD and control cases and the Fund for Scientific Research (FWO) for supporting this research.

\section{Authors' affiliations}

B R J Van Everbroeck, J Boons, P Cras, Department of Neurobiology, Born Bunge Foundation, University of Antwerp, Wilrijk, Belgium

This study was supported by the Fund for Scientific Research (FWO). BVE is a postdoctoral researcher of the Fund for Scientific Research (FWO).

Competing interests: none declared

Correspondence to: B Van Everbroeck, Laboratory of Neurobiology, Born Bunge Foundation (BBS), University of Antwerp, Campus Drie Eiken, Universiteitsplein 1, B-2610 Wilrijk, Belgium; bart. vaneverbroeck@ua.ac.be

Received 7 November 2003

In revised form 19 April 2004

Accepted 19 April 2004

\section{REFERENCES}

1 Masters CL, Harris JO, Gajdusek DC, et al. Creutzfeldt-Jakob disease: patterns of worldwide occurrence and the significance of familial and sporadic clustering. Ann Neurol 1979;5:177-88.
2 Zeidler M. 14-3-3 cerebrospinal fluid protein and Creutzfeldt-Jakob disease. Ann Neurol 2000;47:683-4.

3 Zerr I, Pocchiari M, Collins S, et al. Analysis of EEG and CSF 14-3-3 proteins as aids to the diagnosis of Creutzfeldt-Jakob disease. Neurology 2000;55:811-15.

4 Van Everbroeck B, Quoilin S, Boons J, et al. A prospective study of CSF markers in 250 patients with possible Creutzfeldt-Jakob disease. J Neurol Neurosurg Psychiatry 2003;74:1210-14.

5 Sanchez-Valle R, Saiz A, Graus F. 14-3-3 Protein isoforms and atypical patterns of the 14-3-3 assay in the diagnosis of Creutzfeldt-Jakob disease. Neurosci Lett 2002;320:69-72

6 Peoc'h K, Schroder HC, Laplanche J, et al. Determination of 14-3-3 protein levels in cerebrospinal fluid from Creutzfeldt-Jakob patients by a highly sensitive capture assay. Neurosci Lett 2001;301:167-70.

7 Green AJ. Use of 14-3-3 in the diagnosis of Creutzfeldt-Jakob disease. Biochem Soc Trans 2002;30:382-6

8 Beaudry $P$, Cohen $P$, Brandel JP, et al. 14-3-3 protein, neuron-specific enolase and S-100 protein in cerebrospinal fluid of patients with CreutzfeldtJakob disease. Dement Geriatr Cogn Disord 1999;10:40-6.

9 Chapman T, McKeel DW, Morris JC. Misleading results with the 14-3-3 assay for the diagnosis of Creutzfeldt-Jakob disease. Neurology 2000:55:1396-7.

10 Burkhard PR, Sanchez JC, Landis T, et al. CSF detection of the 14-3-3 protein in unselected patients with dementia. Neurology 2001;56:1528-33.

11 Zerr I, Poser S. Clinical diagnosis and differential diagnosis of CJD and VCJD. With special emphasis on laboratory tests. APMIS 2002;1 10:88-98.

12 Green AJ. Cerebrospinal fluid brain-derived proteins in the diagnosis of Alzheimer's disease and Creutzfeldt-Jakob disease. Neuropathol Appl Neurobiol 2002;28:427-40.

13 Wilffang J, Otto M, Baxter HC, et al. Isoform pattern of 14-3-3 proteins in the cerebrospinal fluid of patients with Creutzfeldt-Jakob disease. J Neurochem 1999:73:2485-90.

14 Van Everbroeck B, Green AJ, Pals P, et al. Decreased levels of amyloid-beta 1-42 in cerebrospinal fluid of Creutzfeldt-Jakob disease patients. J Alzheimer's Dis 1999:1:419-24.

15 Van Everbroeck B, Dobbeleir I, De Waele M, et al. Differential diagnosis of 201 possible Creutzfeldt-Jakob disease patients. J Neurol 2004;251:298-304.

16 Aksamit AJ, Preissner CM, Homburger HA. Quantitation of 14-3-3 and neuron-specific enolase proteins in CSF in Creutzfeldt-Jakob disease. Neurology 2001;57:728-30.

17 Tyler JW, Lakritz J, Weaver D, et al. The 14-3-3 cerebrospinal fluid immunoassay lacks utility in the diagnosis of clinical scrapie. J Vet Diagn Invest 2001;13:537-9.

18 Otto M, Esselmann H, Schulz-Shaeffer W, et al. Decreased beta-amyloid 1-42 in cerebrospinal fluid of patients with Creutzfeldt-Jakob disease. Neurology 2000;54:1099-102. 